\title{
Geometrical form assessment of a CFD based breathing thermal manikins, designed by simplified polygonal shapes
}

\author{
Martin Ivanov ${ }^{1, *}$, and Sergey Mijorski ${ }^{2}$ \\ ${ }^{1}$ Technical University - Sofia, FPEPM, Department:" Hydroaerodynamics and Hydraulic Machines", Sofia 1000, Bulgaria \\ ${ }^{2}$ SoftSim Consult Ltd., Consultant at TU - Sofia, FPEPM, Sofia 1000, Bulgaria
}

\begin{abstract}
The presented paper focuses on a CFD based analyses of the complexity in the geometrical shape of the breathing thermal manikins, associated with their main functionalities. Both impacts of the external manikin's form were studied - over the velocity and over the temperature fields in the thermal plume zone above the head. Three different geometrical shapes are analysed - a physiologically identified (called Humanoid Manikin) and two other shapes, designed to match the overall $95^{\text {th }}$ percentile of the anthropometric size of the standard person (called Polygonal Manikins). The first model represents a comprehensive multifaceted figure of a manikin with high degree of physiological identity with a female human being. The second and third one, are simplified, but still with anatomically realistic component forms, accurately representing the anthropometric size of a standard person. The difference between them is in the presence of additional flow optimization collars in the third model. The numerical results demonstrate the clear impact of the manikins' geometrical characteristics over the simulated breathing and convective flows. The optimization with the proposed collars had a positive effect over the resulted flow acceleration at top head and chest zones. However, the improvement of the flow characteristics was observed for two of the simulated three breathing phases and further shape optimization is required.
\end{abstract}

\section{Introduction}

The use of virtual thermal manikins, particularly at the design stage of the indoor environment, is appropriate alternative to the genuine thermal manikins' experiments [1]. Also, the experimental studies with real thermal manikins are sometimes expensive, time consuming, may require highly skilled labour and are relatively difficult to conduct [2]. However, the need of relatively inexpensive and easy to construct and use real thermal manikins will always exists, considering that all performed simulation studies need to be verified and validated with experimental measurement data.

In today's engineering research practice, the real and virtual thermal manikins are used for overall assessment of the human thermal comfort, as well as for analyses of the indoor air quality in the occupied spaces. They represent modern, highly complex tools for measurement and analyses of the convective flows around human bodies in different conditions, without excessive risk of exposure to the people themselves. Also, they allow simulating different levels of physical activity, as well as some human actions such as breathing, sweating, sneezing, coughing and others [3, 4].

A simplified structure of a virtual thermal manikin with breathing functionality is suggested by the authors in this paper, and the first stage of the breathing flow modelling is presented in [5]. This structure signifies an opportunity for real manikin's production cost optimization. However, a numerical shape optimization at this stage of the design, by means of CFD (Computational Fluid Dynamics) based analyses, is required, in order to assess the overall design performance $[6,7]$

That is why the objective of the presented study is to analyse the geometrical characteristics' impact over the thermal plume and breathing flow phases, generated by the virtual breathing thermal manikins. The main task is to develop and perform simulation studies of the flows from the simulated breathing cycle for three manikin geometries - the physiologically identical one (called Humanoid Manikin) and two proposed simplified shape one (called Polygonal Manikins). The two polygonal manikins differ by the implementation of specially designed collars. The differences or the similarities in the velocity and thermal flow fields between the two shapes will provide initial assessment measure for the both models performance.

\section{Humanoid and Polygonal geometry modelling}

The used Humanoid Manikin is a multifaceted 3D female manikin, specially remodelled and adapted for the purpose of the study. It represents with high degree of physiological identity a real female human, and has an approximate surface area of $1.8 \mathrm{~m}^{2}$ and height of $1.65 \mathrm{~m}$.

\footnotetext{
* Corresponding author: $\underline{m}$ ivanov@tu-sofia.bg
} 
The nasal valve opening was built according to the study of Lin [8] and was initially used by the authors in [5]. The nasal opening area is $7.3 \times 10^{-5} \mathrm{~m}^{2}$, as shown on Fig.1. The normal to the nasal opening was specified to 45 degrees from the vertical body axis. Furthermore, exhaust walls from the nasal valve to the nose end edge were inclined to 15 degrees according to Nilsson [3] and Lin [8].

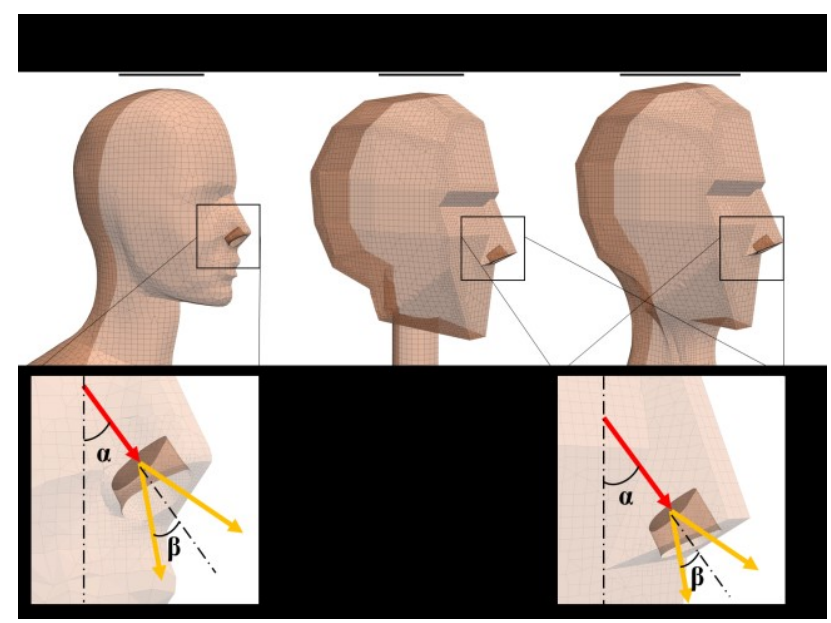

Fig. 1. 3D models and nasal valve geometry details.

The first Polygonal Manikin represents the overall $95^{\text {th }}$ percentile of the anthropometric size of a standard person. It is designed by Georgi Chervendinev (Engineering Design Lab at TU-Sofia, http://design.tusofia.bg), in order to meet the basic requirements of the ergonomic design area. It has an approximate surface area of $2 \mathrm{~m}^{2}$ and height of $1.75 \mathrm{~m}$. The nasal valve opening is constructed in the same way as in the Humanoid Manikin, described above.

The second "Polygonal Manikin with Collars" is in general the same as the first one, but with specially implemented collars in the neck and waist areas. It is supposed that these additional collars will improve the thermal plume characteristics in the free convection zone above the manikin. Both polygonal manikins are proposed for use in real research applications, as well as for educational purposes, complying with the requirements for: high degree of manufacturability; high degree of mobility; availability in different scales and open design.

\section{CFD modelling}

Two different approaches are implemented, for the purpose of the presented study. In Approach 1, the Humanoid Manikin performance is compared with the first Polygonal Manikin, for the three breathing phases: inhalation, free convection (no breathing), and exhalation phase. In Approach 2, the three presented manikin geometries are compared only at the free convection phase, where there is no breathing flow generated. Those two approaches represent the most significant operational situations, at which the geometrical shapes might be compared.

\subsection{Spatial discretization and model setup}

The model discretization in both presented approaches, was made with snappyHexMesh utility, part of an ENGYS ${ }^{\circledR}$ (www.engys.com), an enhanced version of the CFD code OpenFoam $\mathbb{R}$ (www.openfoam.com). The two $3 \mathrm{D}$ models of the thermal manikins were positioned in rectangular shaped room with dimensions: $3 \mathrm{~m}$ height, 4 $\mathrm{m}$ width and $4 \mathrm{~m}$ depth.

In Approach 1, the generated numerical grids were with total of 1140,000 poly-mesh control volume elements for the Humanoid Manikin and 1,417,722 polymesh cells for the Polygonal Manikin. The base cell size was defined to $4 \times 10^{-2} \mathrm{~m}$, for both models. To capture the nasal valve geometrical features, the maximum level of cells refinement reached $6.25 \times 10^{-4} \mathrm{~m}$. The grids are well refined at the surfaces of the manikins, with a first layer height of approximately $0.5 \times 10^{-3} \mathrm{~m}$. The levels of refinement in this Approach 1, were assessed with a detailed analysis of the $y+$ values over the manikin surfaces. In the specified exhale air flow conditions, the $\mathrm{y}+$ values were below 4 to all surface cells of the models (see Fig. 2) as recommended in the work of Spalart [9]. Thus, the models matched the requirements for resolving accurately the turbulent flow over manikins' surfaces in the set of RANS (Reynolds-Averaged Navier-Stokes) CFD simulations.

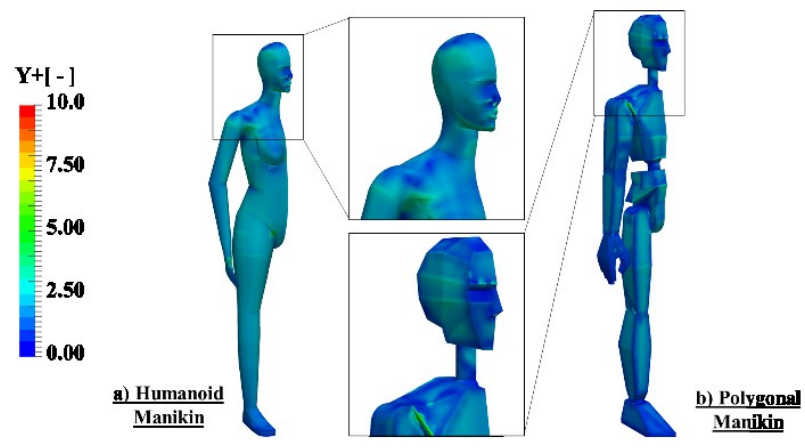

Fig. 2. Y+ fields over manikins' surface.

Two sets of 3D steady state simulations, based on RANS equation method were performed in Approach 1, and total of nine RANS simulations were performed in Approach 2. All three phases of the human breathing cycle were modelled for each of the manikin models, including: inhaling, exhaling and no breathing (the free convection flow case). The solver for the buoyant turbulent flow of incompressible fluids buoyantBoussinesqSimpleFoam with combinations of semi-implicit method for pressure-linked equations (SIMPLE) algorithms was employed for all simulations in both approaches.

In Approach 2, all numerical grids were constructed by following one and the same meshing logic, i.e. defining base cell size to $4 \times 10^{-2} \mathrm{~m}$ and increasing the refinement level to $6.25 \times 10^{-4} \mathrm{~m}$ in the nasal valve zone. This was done, in order to capture the detailed geometrical features of the manikins. Also, the numerical models were again well refined at the surfaces of the manikins, with a first layer height of approximately $0.5 \times 10^{-3} \mathrm{~m}$, as shown above. These 
refinements were again dictated from the requirement for low $\mathrm{y}+$ values over the manikin surfaces, recommended in the work of Spalart [9]. The poly-mesh details for Approach 2 are presented in Table 1.

Table 1. Poly-mesh details.

\begin{tabular}{|c|c|c|c|}
\hline $\begin{array}{c}\text { Type of the } \\
\text { elements }\end{array}$ & $\begin{array}{c}\text { Humanoid } \\
\text { model }\end{array}$ & $\begin{array}{c}\text { Polygonal } \\
\text { model }\end{array}$ & $\begin{array}{c}\text { Polygonal } \\
\text { model with } \\
\text { collars }\end{array}$ \\
\hline hexahedral & $94[\%]$ & $92[\%]$ & $92[\%]$ \\
\hline polyhedral & $3[\%]$ & $6[\%]$ & $6[\%]$ \\
\hline prisms & $2[\%]$ & $2[\%]$ & $2[\%]$ \\
\hline wedges & $<1[\%]$ & $<1[\%]$ & $<1[\%]$ \\
\hline tetrahedral & $<1[\%]$ & $<1[\%]$ & $<1[\%]$ \\
\hline Elements & $\begin{array}{c}1139195 \\
\text { cells }\end{array}$ & $\begin{array}{c}1417722 \\
\text { cells }\end{array}$ & $\begin{array}{c}1413376 \\
\text { cells }\end{array}$ \\
\hline
\end{tabular}

In both approaches, the Shear Stress Transport (SST) $\mathrm{k}-\omega$ turbulence model was applied in the simulations, as suggested in [10]. This is a two-equation eddy-viscosity model, where the SST model formulation combines the use of a k- $\omega$ in the inner parts of the boundary layer, but also switches to a k- $\varepsilon$ behavior in the free-stream regions of the computational domain. Further details of the selected RANS turbulence model can be found in work of Menter [11].

The resolving of the buoyant flow close to the manikins' surface is associated with high nonlinearity of the solutions and there could be small fluctuations in the velocity and pressure fields even under steady state conditions, especially in the more refined zones. To compensate this, a flow averaging at the final 1000 iterations was introduced for each simulation in both approaches. This helped in the smoothing of any small flow fluctuations in the most refined zones of the domains.

\subsection{Initial and boundary conditions}

The material properties of the modelled fluid were selected to match the reference conditions where the pressure was $101,325 \mathrm{~Pa}$ and air temperature was $20^{\circ} \mathrm{C}$. Thus the air density was $1.204 \mathrm{~kg} / \mathrm{m}^{3}$; dynamic viscosity was $1.82 \times 10^{-5} \mathrm{~kg} /(\mathrm{m} . \mathrm{s})$; kinematic viscosity was $1.51 \mathrm{x}$ $10^{-5} \mathrm{~m}^{2} / \mathrm{s}$ and specific heat was $1006 \mathrm{~J} /(\mathrm{kg} \cdot \mathrm{K})$.

In Table 2 and Fig. 3 are given all the different boundary conditions adopted in the CFD simulations for both approaches, together with the associated heat fluxes from the thermal manikins. The heat fluxes were derived from the study of Nilsson [3], based on total heat release rate of $110 \mathrm{~W}$ for the whole manikin surface.

The nasal valve openings were specified as velocity inlet for exhale phase and outlet for the inhale. The flow rates were calculated based on the study of Lin [8]. The total flow rates for both inhaling and exhaling were approximated as follows:

$$
\mathrm{Q}=(\Delta \mathrm{P} . \pi . \mathrm{r} 4) /(8 . \eta . \mathrm{L})
$$

where:

- $\mathrm{Q}$, total flow rate during inhaling and exhaling, $\mathrm{m}^{3} / \mathrm{s}$;
- $\Delta \mathrm{P}$, pressure in the range of $40-80 \mathrm{~Pa}$, selected value of $60 \mathrm{~Pa}$;

- L, approximated nasal valve length, $0.33 \mathrm{~m}$;

- $\mathrm{r}$, approximated nasal valve radius, $0.11 \mathrm{~m}$;

- $\eta$, kinematic viscosity, $\mathrm{m}^{2} / \mathrm{s}$;

- $\eta 20, \eta$ at $20^{\circ} \mathrm{C}, 1.51 \times 10^{-5} \mathrm{~m}^{2} / \mathrm{s}$;

- $\eta 36, \eta$ at $36{ }^{\circ} \mathrm{C}, 1.66 \times 10^{-5} \mathrm{~m}^{2} / \mathrm{s}$.

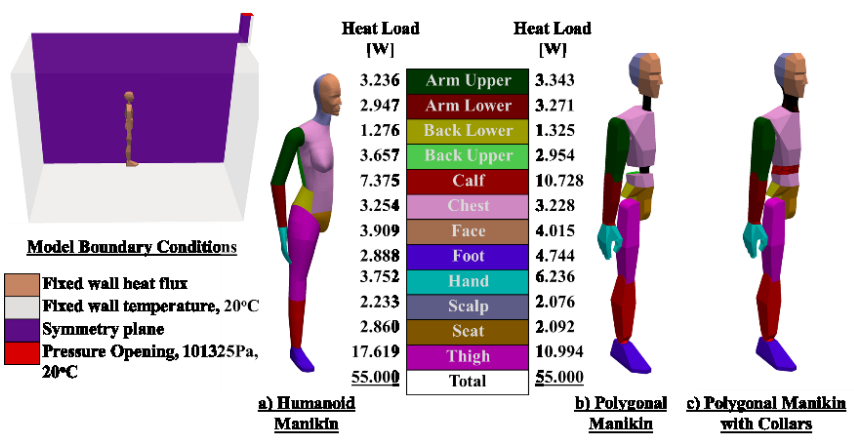

Fig. 3. Initial and boundary conditions.

The Reynolds number for the nasal flow was approximated based on the eq. 2 .

$$
\mathrm{Re}=2 . \mathrm{r} . \mathrm{Q} \cdot \rho / \eta
$$

This way the turbulent intensity for the velocity inlet was calculated to $6.8 \%$.

Table 2. Implemented boundary conditions.

\begin{tabular}{|c|c|c|c|c|}
\hline $\begin{array}{c}\text { Boundary } \\
\text { Name }\end{array}$ & $\begin{array}{c}\text { Boundary } \\
\text { Conditions }\end{array}$ & Inhale & $\begin{array}{c}\text { Free } \\
\text { convection } \\
\text { flow }\end{array}$ & Exhale \\
\hline $\begin{array}{c}\text { No Slip } \\
\text { Walls }\end{array}$ & $\begin{array}{c}\text { Surface } \\
\text { temperature, } 20 \\
{\left[{ }^{\circ} \mathrm{C}\right]}\end{array}$ & Yes & Yes & Yes \\
\hline $\begin{array}{c}\text { Vent } \\
\text { Opening }\end{array}$ & $\begin{array}{c}\text { Air temperature } \\
\text { and pressure, } 20 \\
{\left[{ }^{\circ} \mathrm{C}\right] \text { and } 101325} \\
{[\mathrm{~Pa}]}\end{array}$ & Yes & Yes & Yes \\
\hline Manikin & $\begin{array}{c}\text { Fixed heat flux } \\
\text { as per Fig.3 }\end{array}$ & Yes & Yes & Yes \\
\hline $\begin{array}{c}\text { Nasal valve } \\
\text { inlet }\end{array}$ & $\begin{array}{c}\text { Inlet flow rate, } \\
6.29 \times 10^{-4} \\
{\left[\mathrm{~m}^{3} / \mathrm{s}\right] \text { at } 36\left[{ }^{\circ} \mathrm{C}\right]}\end{array}$ & No & No & Yes \\
\hline $\begin{array}{c}\text { Nasal valve } \\
\text { outlet }\end{array}$ & $\begin{array}{c}\text { Outlet flow rate, } \\
6.91 \times 10^{-4} \\
{\left[\mathrm{~m}^{3} / \mathrm{s}\right]}\end{array}$ & Yes & No & No \\
\hline Symmetry & $\begin{array}{c}\text { Symmetry plane } \\
\text { Symmes }\end{array}$ & Yes & Yes & Yes \\
\hline
\end{tabular}

\section{Numerical results and discussion}

The numerical results in Approach 1 are presented in from of velocity fields for the exhale phase of the flow. Two different sections from the solutions are extracted. The first is a perpendicular section to the symmetry plane of the domain and the second is at the symmetry plane. Additionally, it is presented a pointwise data in graphical format, at a horizontal profile above the 
manikins' head and parallel to the symmetry plane at 2.5 $\mathrm{m}$ height from the floor.

It is seen a visible difference in the velocity fields in both sections from the solutions. The resulted thermal plume in the case of Polygonal Manikin is with lower velocity and it is more diffused above the head with wider shape of the flow (see Fig.4). The maximum velocity measured in the Humanoid Manikin free convection flow model was $0.6 \mathrm{~m} / \mathrm{s}$, while in the Polygonal this value dropped to $0.54 \mathrm{~m} / \mathrm{s}$.

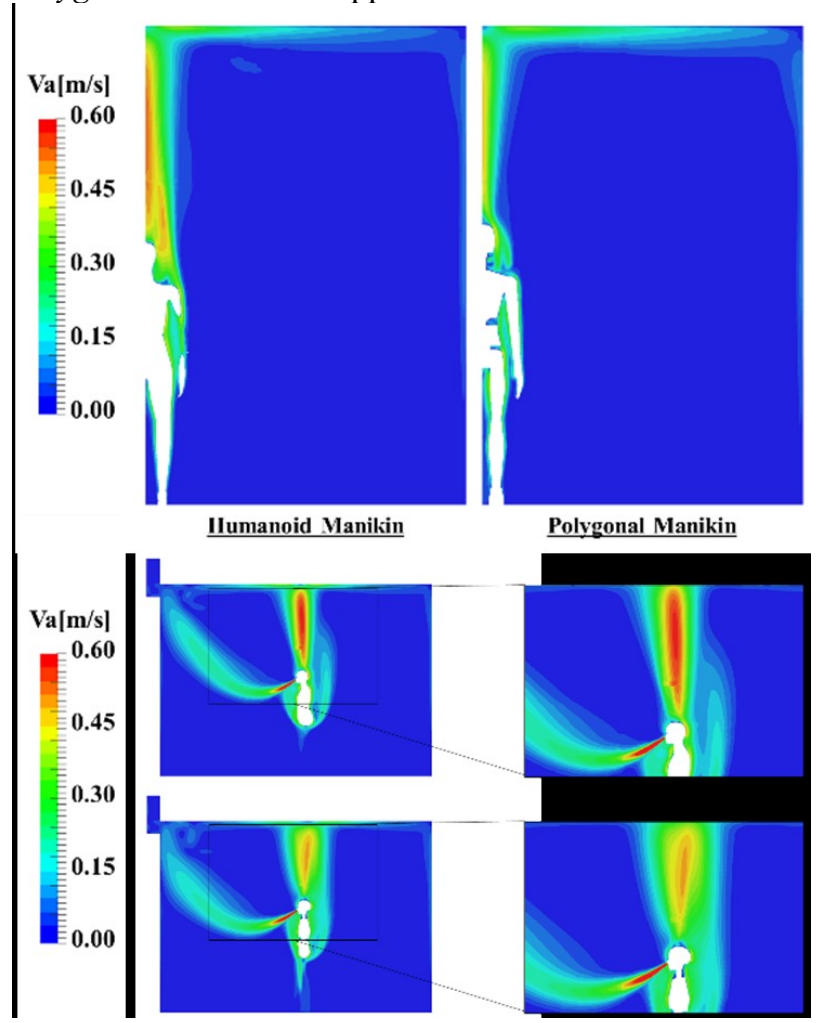

Fig. 4. Velocity fields in Approach 1 - exhale breathing phase.

The observation regarding the shape of the plume can be confirmed also from the horizontal graphical data in Fig.5, where the profile of Humanoid Manikin is with more distinguished peak at the centre, while in the polygonal model is more outspread with smoother gradients at the end from the back side of the manikin.

The increased separations of the free convective flow along the Polygonal Manikins are the main driving force for the noticeable differences in the illustrated comparative plots. The polygonal structures of the Polygonal Manikins have resulted in sharp edges at all body parts. Together with the mobility feature, which required recess zones and many additional openings, it leads to recirculation zones and stronger flow separations compared to the Humanoid Manikin model. The effect of such zones is more strongly observed at the recess below the head and at the big gap between upper and lower backs for the Polygonal Manikins.

The numerical results in Approach 2 are presented again at the same two different sections from the velocity field solutions, for the free convection flow (no breathing phase) of the breathing cycle (see Fig.6 and Fig.7). Again, a pointwise data for all three phases is presented in graphical format, for a horizontal profile above the manikins' head, again parallel to the symmetry plane at $2.5 \mathrm{~m}$ height from the floor.
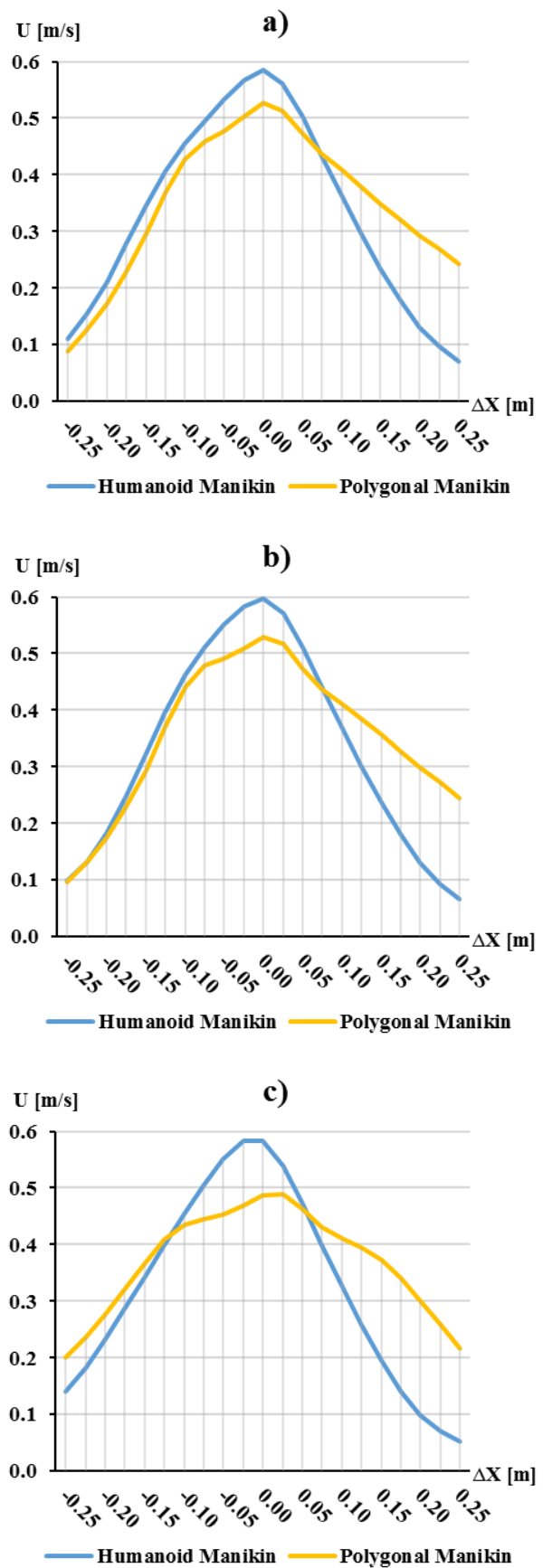

Fig. 5. Horizontal air velocity at monitoring points in Approach 1: a) Inhale; b) Free convection; c) Exhale.

The monitoring data of the numerical solutions in Approach 2, has shown again that the maximum air velocity for the free convection flow is reaching the value of $0.6 \mathrm{~m} / \mathrm{s}$ for the Humanoid Manikin, while the other two models have resulted in lower velocities. For the Polygonal Manikin models, it was registered a maximum velocity of $0.54 \mathrm{~m} / \mathrm{s}$ and $0.57 \mathrm{~m} / \mathrm{s}$ for the optimized model with the collars.

As it can be seen on Fig. 6 and Fig.7, the thermal plume of the Humanoid Manikin is well formed, with higher acceleration in close proximity of the manikin head and as well in the chest zone. Also, there is a clear 
flow separation at the manikin's back zone resulted from the increased flow acceleration at the legs (see Fig. 6a and Fig. 7a).

Again, the polygonal models are associated with increased turbulence of the free convective flow and better mixing of the flow at the manikin's back zone (see Fig. $6 \mathrm{~b}$ and Fig. 7b). It is confirmed that, this effect is in result of the many mechanical openings and the sharp edges of the polygonal elements at the legs and lower back.

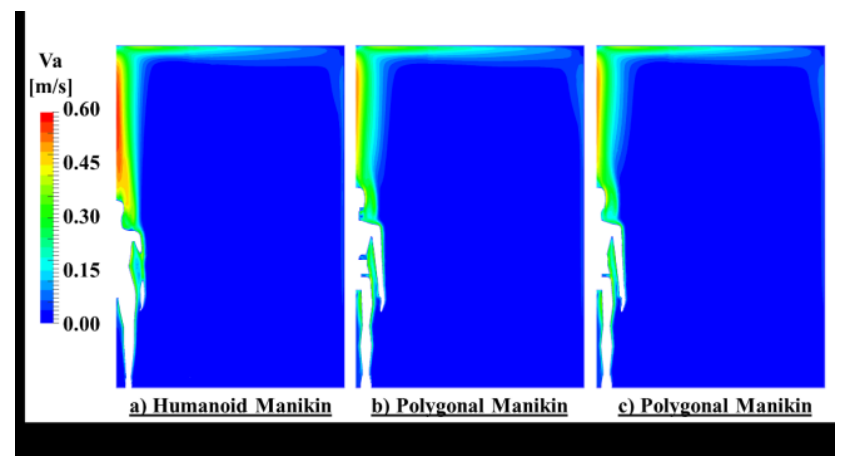

Fig. 6. Velocity fields for free convection (no breathing phase) $-\mathrm{X}$ section.

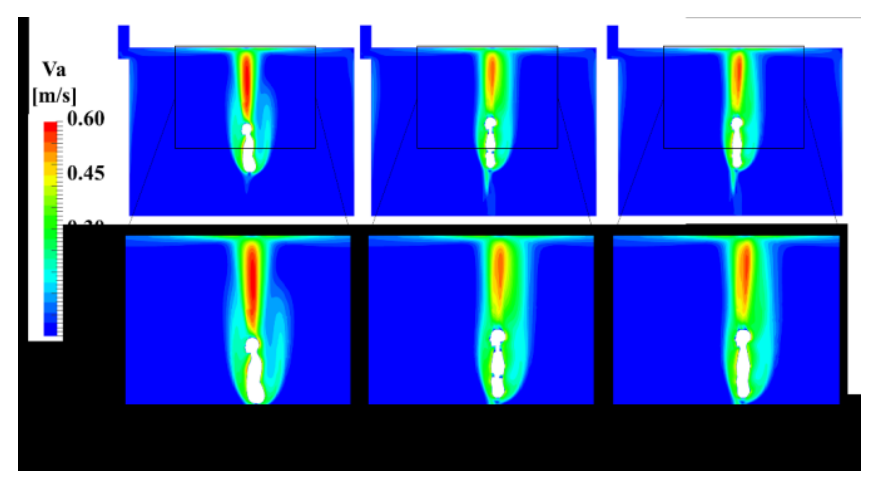

Fig. 7. Velocity fields for free convection (no breathing phase) - Y section.

The polygonal model optimization, with the introduction of the two collars, has resulted in visible flow improvements along the manikin surfaces, but the impact of the increased flow mixing at the legs and lower back zones is still visible, as shown in Fig. 6c and Fig. 7c. The manikin's back flow is well mixed without distinct flow separation, which was observed in the Humanoid Manikin. The big recirculation zones at the neck and the waist do not exist anymore and there is a visible improvement of the flow acceleration near the chest.

The pointwise data showed in Fig. $8 \mathrm{a}$ and b, clearly illustrates the flow characteristics improvement, resulted from the introduction of the two collars. However, for the exhale phase results in Fig. 8c, it was observed worsening of the thermal plume profile. The impact of the exhale flow increases with proposed modifications. Also, from the graphical data is visible the need of further polygonal model modifications in the back and leg zones. Furthermore, the shapes of the velocity profiles are different, especially in the front zone of the manikins.
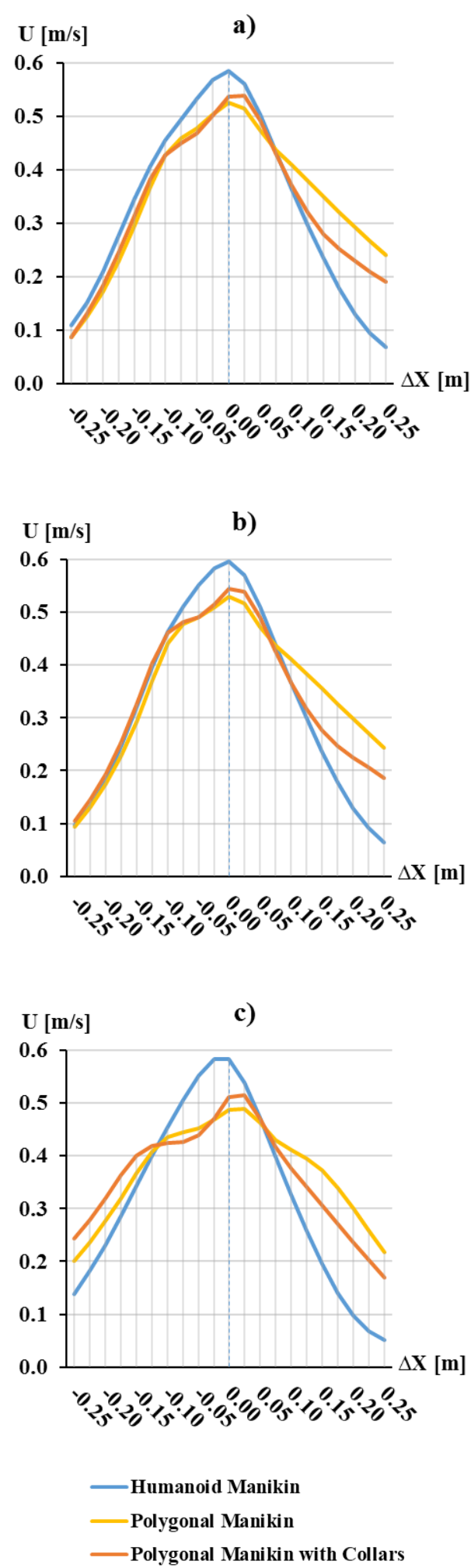

Fig. 8. Horizontal air velocity at monitoring points: a) Inhale; b) Free convection; c) Exhale.

\section{Conclusion}

The comparative analysis of the numerical results in both presented approaches, has illustrated several weaknesses of the proposed Polygonal Manikin model. Sharp edges 
and big recess zones significantly alters the free convection flow compared to the Humanoid Manikin. The recirculation zones and increased flow separation lead to wider thermal plume above the head of the manikin with lower air velocity.

The proposed geometrical optimization has led to improvement of the free convection flow along the proposed Polygonal Manikin. Also, the analyses of the results assisted the understanding of the implications caused by the geometrical characteristic of the model. However, the comparative analyses between smooth surface manikin and polygonal with many sharp edges and openings by itself is a difficult task. The Humanoid Manikin model would be characterized with more laminar flow along the surfaces, while the polygonal with more turbulent flow and mixing at the upper zones of the model. In reality, the human body is covered with clothing and accessories (as hat, headphones, glasses and etc,), which would result as well in more turbulent flow and better mixing, which then could have a better match in the thermal plume characteristics of the proposed polygonal manikin.

Further work of the Polygonal Manikin optimization should be taken in direction of introduction of the postmanufacture filleting of the sharp edges and additional closing of the mechanical openings with highly flexible material, which will not restrict the movability feature of the model. Furthermore, numerically derived results should be compared with experimental data of clothed thermal manikin. This way, a validation of the proposed virtual thermal manikin would be accomplished and the next design stages for the breathing system could be taken.

The presented study is supported by "RDS" at TU-Sofia, as part of the activities under the "Perspective leaders" project, with Contract № 181ПР0005-02, entitled: "Experimental comparison of the air jet characteristics during exhalation, with numerical results from developed virtual breathing thermal manikin".

\section{References}

[1] M. Ivanov, "Compact Breathing Simulation System, Developed as Additional Functionality for Thermal Manikins", "Romanian Journal of Building Services", Vol.1, No.3, ISSN: 2393-5154, pp. 1-12, 2015;

[2] T. Madsen, "Development of a breathing thermal manikin", Proceedings of the 3rd international meeting on thermal manikin testing 3IMM, Stockholm, Sweden, 12-13 October 1999;

[3] H. Nilsson, "How to Build and Use a Virtual Thermal Manikin Based on Real Manikin Methods", Sixth International Thermal Manikin and Modelling Meeting", "Thermal Manikins and Modelling", ISBN: 962-367-534-8, 2006;

[4] E. Bjørn, „Simulation of human respiration with breathing thermal manikins", Proceedings of the 3rd international meeting on thermal manikin testing 3IMM, Stockholm, Sweden, 12-13 October 1999;
[5] M. Ivanov, S. Mijorski, "CFD modelling of flow interaction in the breathing zone of a virtual thermal manikin", "Energy Procedia" Journal, Volume 112, pp. 240-251, ISSN: 1876-6102, Elsevier, 2017;

[6] M. Ivanov, S. Mijorski, „Geometrical characteristics' impact over the thermal plume modelling with breathing thermal manikins", 15th International Conference on Environmental Science and Technology, ISSN 11065516, Manuscript ID: cest2017_00414, Rhodes, Greece, 31 August to 2 September 2017;

[7] M. Ivanov, S. Mijorski, "Numerical optimisation of polygonal breathing thermal manikins", Proceedings of: "6th International Conference on Thermal Equipment, Renewable Energy and Rural Development - TE-RE-RD 2017", Moieciu de Sus - Romania, Politehnica Press, ISSN 2457 - 3302, pp. 63-68, June 2017;

[8] S. Lin, "Nasal Aerodynamics", Chief Editor: Arlen D Meyers, MD, MBA, http: //emedicine. medscape.com/article/874822-overview\#a1, Updated: May 04, 2018;

[9] P. Spalart, Young-person's guide to detached-eddy simulations grids, NASA/CR-2001-21103, Boeing Commercial Airplanes, Seattle, Washington, 2001;

[10] F. Menter, "Zonal Two Equation k- $\omega$ Turbulence Models for Aerodynamic Flows", AIAA Paper 93-2906, 1993;

[11] F. Menter, "Turbulence Modelling for Engineering Flows", ANSYS Inc., 2011; 